\title{
Documento de consenso sobre el uso de iSGLT2 en pacientes con enfermedad renal crónica y diabetes
}

\author{
Ricardo Correa-Rotter ${ }^{1 *}$, Juan Rosas-Guzmán²,3, Antonio Méndez-Durán ${ }^{4}$, Mario A. Sebastián-Díaz \\ Odette Del C. Díaz-Avendaño ${ }^{6}$, Roopa Mehta-Pravin y Marco A. Alcocer-Gamba 8,9 \\ ${ }^{1}$ Departamento de Nefrología y Metabolismo Mineral, Instituto Nacional de Ciencias Médicas y Nutrición Salvador Zubirán, Ciudad de México; ${ }^{2}$ Instituto \\ de Diabetes AC en Celaya, Celaya, Guanajuato; ${ }^{3}$ Centro de Especialidades Médicas de Celaya, Celaya, Guanajuato; ${ }^{4}$ Coordinación de Programas \\ Médicos Nivel Central, Instituto Mexicano del Seguro Social (IMSS), Ciudad de México; ${ }^{5}$ Servicio de Nefrología, Hospital Central Sur de Alta \\ Especialidad, Petróleos Mexicanos (PEMEX), Ciudad de México; ${ }^{6}$ Servicio de Nefrología y Trasplante Renal, Hospital Centro Médico Nacional 20 \\ de Noviembre, Instituto de Seguridad y Servicios Sociales de los Trabajadores del Estado (ISSSTE), Ciudad de México; ${ }^{7}$ Unidad de Investigación

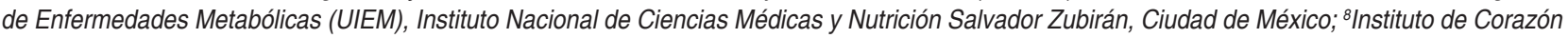 \\ de Querétaro, Querétaro, Querétaro; ${ }^{9}$ Facultad de Medicina, Universidad Autónoma de Querétaro, Querétaro, Querétaro. México
}

\section{Resumen}

La enfermedad renal crónica (ERC) del paciente diabético es frecuentemente una consecuencia directa de la diabetes mellitus (DM) de larga evolución y se la conoce como nefropatía diabética. En México cerca del 50\% de los pacientes en terapia sustitutiva de la función renal tienen ERC por DM, y este porcentaje podría aumentar en los próximos años. Nuevas opciones terapéuticas, combinadas con cambios en el estilo de vida, han mejorado el control de la glucemia y pueden contribuir sustancialmente a retrasar la aparición o la progresión a estadios avanzados de la ERC. Las sociedades científicas internacionales han elaborado guías clínicas para el diagnóstico y manejo de la nefropatía diabética, sin embargo, en algunos puntos estas recomendaciones no se adaptan a la realidad mexicana. Se presentan las conclusiones de un consenso realizado por especialistas mexicanos sobre diabetes y ERC, con especial énfasis en el uso de los inhibidores del cotransportador de sodio-glucosa.

PALABRAS CLAVE: Enfermedad renal crónica. Nefropatía diabética. Diabetes tipo 2. Recomendaciones. iSGLT2.

\section{Consesus document in the use of SGLT inhibitors in patients with diabetes and chronic kidney disease}

\begin{abstract}
Chronic kidney disease (CKD) in the diabetic patient is mainly a consequence of long-term diabetes mellitus itself. In Mexico approximately $50 \%$ of patients on dialysis are diabetics and this will could increase in the coming years. New therapeutic options available, combined with lifestyle changes, have improved glycemic control and may contribute to delay the onset as well as the progression of CKD. International scientific societies have developed clinical guidelines for the diagnosis and management of CKD in diabetics, although in some points, these recommendations are not adapted to the Mexican reality. We hereby present the conclusions of the consensus reached by Mexican specialists on diabetic nephropathy.
\end{abstract}

KEYWORDS: Chronic kidney disease. Type 2 diabetes. Recommendations. iSGLT2.

Correspondencia:

*Ricardo Correa-Rotter

E-mail: correarotter@gmail.com
Fecha de recepción: 20-10-2021

Fecha de aceptación: 27-10-2021

DOI: 10.24875/GMM.M21000595
Gac Med Mex. 2022;158(M2):M1-12

Disponible en PubMed

www.gacetamedicademexico.com CC BY-NC-ND (http://creativecommons.org/licenses/by-nc-nd/4.0/). 


\section{Introducción}

La enfermedad renal crónica (ERC) en el paciente diabético tipo 2 generalmente es consecuencia directa de la diabetes mellitus (DM) de larga evolución, sin embargo, en algún porcentaje de la población con esta enfermedad también influyen o pueden ser condicionantes de ERC otros factores de daño renal como la hipertensión arterial misma y en mucho menor grado, la coexistencia de otras enfermedades glomerulares primarias ${ }^{1,2}$. La ERC atribuible a la DM, o nefropatía diabética (ND), ocurre en el $20-40 \%$ de las personas con diabetes de más de 15 años de evolución ${ }^{3}$. En México, la ND corresponde a la causa de tratamiento dialítico de alrededor del $50 \%$ de los pacientes ${ }^{2}$. Durante las últimas décadas se ha observado un incremento de la incidencia de la ERC en personas con DM tipo 1 (DM1) y tipo 2 (DM2), debido principalmente al incremento de la obesidad tanto infantil como en adultos, al sedentarismo y a la hipertensión, así como al aumento de la esperanza de vida ${ }^{4}$.

Un estudio observacional realizado en México entre 1998 y 2014, en el que se tomó como base un registro nacional y que utilizó como metodología de análisis el data mining, describe una prevalencia de ERC secundaria a diabetes de aproximadamente el $48 \%{ }^{5}$. Estas cifras son algo superiores a las publicadas por la ESC (Sociedad Europea de Cardiología), que indican que aproximadamente el $30 \%$ de los pacientes con DM1 y el $40 \%$ con DM2 desarrollarán ND ${ }^{6}$.

Se calcula que más de 450 millones de personas en todo el mundo padecen DM, lo que representa más del $6.5 \%$ de la población, y estas cifras se incrementarán en los próximos años ${ }^{7}$. La misma tendencia al alza se observa en la población mexicana, ya que según los datos de la Encuesta Nacional de Salud y Nutrición de Medio Camino del año 2018 ${ }^{8}$, la prevalencia conocida de la DM se incrementó del $4.6 \%$ en adultos en el año 2000 al $10.4 \%$ en el año 2018; y se calcula que, de no tomarse medidas relevantes, en el año 2045 estas cifras podrían llegar hasta el 20\% de la población mexicana ${ }^{8,9}$.

Se debe tener presente que la hipertensión arterial, la obesidad y la diabetes per se son factores que aumentan la mortalidad cardiovascular (CV), así como el riesgo de desarrollo de ERC, lo que a su vez provoca un incremento en los costos de atención ${ }^{10}$; en México, el Instituto Mexicano del Seguro Social en 2018 atendió a 3.5 millones de pacientes con diabetes, en los cuales invirtió un poco más de 37 mil millones de pesos, constituyendo el mayor gasto en salud de ese instituto ${ }^{11}$.

Gracias a nuevas opciones farmacológicas desarrolladas en los últimos años, se han mejorado las opciones de control de la glucemia de los pacientes con DM y, de esta forma, la posibilidad de prevenir el desarrollo o al menos retrasar la progresión de la $\mathrm{ERC}^{4}$. Sin embargo, es fundamental que todas las medidas farmacológicas se acompañen siempre de la implementación de medidas de mejora del estilo de vida o hábitos de vida saludable, en particular evitar obesidad, sedentarismo, tabaquismo y promover el ejercicio periódico, así como una dieta saludable y equilibrada.

\section{Definición de ERC}

Las guías KDIGO (Kidney Disease: Improving Global Outcomes) del año 2020 definen la ERC como la presencia de anomalías estructurales o funcionales del riñón durante al menos tres meses que son no reversibles y la clasifican en distintas categorías de evolución y gravedad, así como pronósticas, según la estimación de la tasa de filtrado glomerular estimada (TFGe) y el grado de albuminuria presente ${ }^{4}$. En este sentido, coinciden con las guías de la ESC, que a su vez recomienda la determinación de microalbuminuria con el fin de identificar a personas con diabetes que estén en riesgo de desarrollar alteraciones renales y/o $\mathrm{CV}^{6}$.

Así, los niveles de ERC considerados por las guías KDIGO son:

- ERC leve (nivel G2) cuando la TFGe = $60-89 \mathrm{ml} / \mathrm{min} / 1.73 \mathrm{~m}^{2}$ de superficie corporal (sc).

- ERC de leve a moderada (G3a) si TFGe $=45$ $59 \mathrm{ml} / \mathrm{min} / 1.73 \mathrm{~m}^{2} \mathrm{sc}$.

- ERC de moderada a grave (G3b) si TFGe $=30$ $44 \mathrm{ml} / \mathrm{min} / 1.73 \mathrm{~m}^{2} \mathrm{sc}$.

- ERC grave (G4) cuando la TFGe $=15-29 \mathrm{ml} / \mathrm{min} /$ $1.73 \mathrm{~m}^{2} \mathrm{sc}$.

- Fallo renal (G5) si TFGe $<15 \mathrm{ml} / \mathrm{min} / 1.73 \mathrm{~m}^{2}$ $\mathrm{Sc}^{4,12}$.

\section{Prevención}

La prevención y el diagnóstico temprano de complicaciones microvasculares ocasionadas por la DM son esenciales. Deben ser realizados sistemáticamente por el médico de primer contacto, por médicos internistas y por endocrinólogos. Esta evaluación, 
vigilancia y manejo debería hacerse desde etapas tempranas de la evolución de la diabetes misma e incluir una evaluación multifactorial que comprenda el seguimiento de los cambios en el estilo de vida (dieta, pérdida de peso, control de comorbilidades como hipertensión, hiperlipidemia y cardiopatía isquémica, entre otros), el control glucémico periódico, la determinación de niveles de albuminuria y la estimación de la TFGe mediante la determinación de creatinina sérica y la aplicación de la fórmula CKD-Epi (Chronic Kidney Disease Epidemiology Collaboration). La frecuencia debe estar determinada por la comorbilidad, el nivel de daño a órgano blanco y el estadio de la función renal, pudiendo ser cada año en etapas tempranas y sin evidencia de daño renal o bien cada seis o hasta cada tres meses cuando ya se detecta algún grado de afección $n^{4,13}$.

Recomendaciones

- Se recomienda promover intensamente un apropiado estilo de vida, así como control glucémico frecuente y apropiado (con evaluación cada dos a cuatro meses) con el fin de alcanzar, lo más rápidamente posible, los objetivos terapéuticos marcados y evitar las complicaciones microvasculares y macrovasculares.

- Es recomendable el control anual de albuminuria y de TFGe, para detectar tempranamente la aparición de ND, independientemente del tiempo de evolución de la diabetes.

\section{Tratamiento farmacológico de la diabetes}

Tanto la American Diabetes Association (ADA) como la European Association for the Study of Diabetes (EASD) recomiendan un enfoque centrado en el paciente para elegir el tratamiento farmacológico adecuado.

Este enfoque deberá tener en cuenta los siguientes factores:

- Comorbilidades importantes como la enfermedad cardiovascular aterosclerótica (ASCVD) e indicadores de alto riesgo de ASCVD, ERC e insuficiencia cardiaca (IC).

- Riesgo de hipoglucemia.

- Efectos sobre el peso corporal.

- Efectos secundarios.

- Costo de los fármacos a elegir.

- Tipo de DM (DM1 o DM2).

- Preferencias del paciente ${ }^{14}$.

Existen distintos tipos de fármacos para el control de la glucosa en el paciente con DM:
- Sensibilizadores de insulina (metformina y pioglitazona).

- Estimuladores de la liberación de insulina (sulfonilureas y meglitinidas).

- Insulina en sus diferentes presentaciones.

- Terapias basadas en incretinas (agonistas del receptor del péptido 1 similar al glucagón. [ARGLP $^{1}$ e inhibidores de la dipeptidil peptidasa-4 $\left[\mathrm{iDPP}^{4}\right)$.

- Inhibidor de la absorción de glucosa gastrointestinal (acarbosa).

- Inhibidores de la reabsorción de glucosa en túbulo proximal renal (inhibidores del cotransportador sodio-glucosa tipo 2 [iSGLT ${ }^{2}$ ).

Los iSGLT2 han sido la última clase de agentes antidiabéticos en recibir la aprobación de la Food and Drug Administration (FDA) ${ }^{6,15}$.

\section{Manejo del paciente con diabetes mellitus y enfermedad renal crónica}

El manejo de los pacientes con diabetes y ND es claramente más complejo que el del diabético sin ERC y debe ser multidisciplinario.

El paciente con ERC y DM tiene un riesgo aumentado tanto de complicaciones agudas (hipoglucemia y cetoacidosis) como crónicas, así como la inherente posibilidad de evolución de la enfermedad renal hacia estadios avanzados que pudieran requerir diálisis o trasplante. Adicionalmente, es claro que el paciente con ND, incluso en estadios relativamente tempranos, tiene un riesgo mayor de complicaciones CV que población de edad similar no diabética e incluso que población de edad similar con otras nefropatías ${ }^{4,6}$. Por ello, la selección del esquema farmacológico en cada paciente para el control de la hipoglucemia se deberá basar en: tipo de DM, objetivos terapéuticos específicos, la edad y todos los factores de riesgo identificados en el paciente mismo ${ }^{13}$.

\section{Control glucémico}

Estudios diversos han demostrado que el control glucémico puede reducir el riesgo de progresión de la enfermedad renal tanto en DM1 como en $\mathrm{DM}^{6}{ }^{6}$. Sin embargo, es importante recordar que este control pudiera ser más complejo en el paciente con ND establecida, ya que la reducción de la TFGe implica ajustes tanto en el tipo de antidiabético utilizado como en la dosis de este ${ }^{6}$. 
A pesar de que los objetivos de hemoglobina glucosilada (HbA1c) deberán individualizarse para cada paciente, existen ciertas recomendaciones por parte de las distintas guías terapéuticas, y en general los niveles de $\mathrm{HbA1c}$ que alcanzar son un poco más altos que en pacientes diabéticos sin daño renal. Así, las guías KDIGO recomiendan niveles de $\mathrm{HbA1c}$ entre el 6.5 y $8 \%$ en pacientes con ERC no sometidos a diálisis ${ }^{4}$. De la misma forma se posiciona la American Association of Clinical Endocrinologists and American College of Endocrinology (AACE/ACE) en su consenso del año 2020, en el que se recomienda alcanzar niveles de $\mathrm{HbA} 1 \mathrm{C}>6.5 \%$ en pacientes con enfermedad renal avanzada o con complicaciones macrovasculares ${ }^{13}$.

Un hecho relevante es que se ha demostrado que la variabilidad de la glucosa plasmática en ayunas es un importante predictor de muerte por cualquier causa, por lo que debe ser considerada ${ }^{6}$. Los fármacos que reducen las excursiones de glucosa posprandial, incluidos los AR-GLP1, los iDPP4 y los iSGLT2, han demostrado ser de utilidad para reducir esta variabilidad $^{6}$.

El tratamiento inicial recomendado en todas las guías de práctica clínica más relevantes se centra en la modificación saludable del estilo de vida y la administración temprana de metformina (siempre que no existan contraindicaciones específicas para su uso). En caso de no alcanzar los objetivos glucémicos se recomienda el inicio de tratamiento farmacológico combinado.

En cuanto al nivel de función renal y el uso de metformina, las guías europeas indican el beneficio y la seguridad del empleo de este fármaco en pacientes con ERC en estadios 1 a 3 a (nefropatía leve a moderada), mientras que se deberá ser cauto con su uso en pacientes con TFGe de $31-45 \mathrm{ml} / \mathrm{min} / 1.73 \mathrm{~m}^{26}$. De igual manera se posiciona la AACE, agrupación que recomienda no utilizar metformina en pacientes con TFGe $<45 \mathrm{ml} / \mathrm{min} / 1.73 \mathrm{~m}^{213}$.

Las guías KDIGO indican que el control glucémico en DM1 se centra en el empleo de insulina, mientras que refuerzan el uso de metformina y agregan el uso indicado de iSGLT2 en primera línea para la DM2 cuando la TFGe $\geq 30 \mathrm{ml} / \mathrm{min} / 1.73 \mathrm{~m}^{2}$, y la adición de otro fármaco si fuera necesario para el control glucémico apropiado (AR-GLP1, iDPP-4, insulina, sulfonilureas u otro) ${ }^{4}$.

Las guías ESC recomiendan el uso de iSGLT2 en pacientes con TFGe entre $30-90 \mathrm{ml} / \mathrm{min} / 1.73 \mathrm{~m}^{2}$, ya que estos fármacos, en adición a su efecto hipoglucemiante moderado, se asocian a protección CV y a un menor riesgo de progresión de enfermedad renal ${ }^{6}$. Por otra parte, cuando el paciente ha progresado a requerir terapia dialítica, es preferible el uso de insulina en lugar de antidiabéticos orales ${ }^{4,6}$. Finalmente, y en línea con lo comentado previamente, las guías ADA del 2022 recomiendan el uso de iSGLT2 o de AR-GLP-1 en adición a metformina en pacientes con enfermedad CV (ECV) establecida, IC o ND con TFGe entre $30-60 \mathrm{ml} / \mathrm{min} / 1.73 \mathrm{~m}^{2} \mathrm{sc}^{14}$.

\section{EFICACIA DE LOS ISGLT2 PARA EL CONTROL DE LA GLUCEMIA}

Los iSGLT2 aumentan la excreción renal de glucosa y reducen así moderadamente los niveles elevados de la glucosa en sangre en pacientes con DM2. No están indicados en DM 1. Existen en México tres iSGLT2 comercializados: canagliflozina, dapagliflozina y empagliflozina ${ }^{12,16}$.

La eficacia hipoglucemiante de los iSGLT2 se debe a la inhibición de la reabsorción de la glucosa por parte de los SGLT2; esta inhibición provoca un incremento de la glucosuria, mecanismo que es independiente de la secreción y de la acción de la insulina. Los iSGLT2 también provocan diuresis osmótica y natriuresis. El efecto hipoglucemiante de los iSGLT2, evaluado por la reducción de la $\mathrm{HbA1c}$, es dependiente del nivel de TFGe, de forma que la $\mathrm{HbA1c}$ se reduce un $0.7-0.8 \%$ cuando la función renal es nor$\mathrm{mal}$, un $0.3-0.4 \%$ con TFGe entre $30-60 \mathrm{ml} / \mathrm{min} / 1.73 \mathrm{~m}^{2}$ y no hay efecto de reducción de HbA1c si la TFGe es $<30 \mathrm{ml} / \mathrm{min} / 1.73 \mathrm{~m}^{2}{ }^{12}$. En un estudio publicado por Polidori et al., la canagliflozina redujo la glucosa posprandial por su efecto adicional de retrasar la absorción intestinal de glucosa, lo que permite suprimir las excursiones posprandiales ${ }^{17}$. Por otra parte, se ha demostrado también que aumenta la secreción del péptido 1 tipo glucagón (GLP-1), en adición a su efecto glucosúrico ${ }^{18}$.

Un metaanálisis reciente que evalúa la eficacia y seguridad de los iSGLT2 en pacientes con DM2 y ERC (definida como TFGe $<60 \mathrm{ml} / \mathrm{min} / 1.73 \mathrm{~m}^{2}$ ), y que incluyó 27 estudios que aportaron datos de 7,363 pacientes, observó que los iSGLT2 reducen la $\mathrm{HbA} 1 \mathrm{c}$ (-0.29\%; intervalo de confianza del 95\% [IC 95\%]: -0.39 a -0.19), así como el peso corporal, la presión arterial y la albuminuria. Además, también observó que el tratamiento con iSGLT2 atenuaba el declive anual de la TFGe y reducía el riesgo de aparición de 
complicaciones CV y deterioro de la función renal en pacientes con DM2 y ERC ${ }^{19}$.

\section{Efecto de los iSGLT2 en la glucemia posprandial}

Lograr el control glucémico de ayuno y posprandial es muy relevante para llegar a metas de $\mathrm{HbA} 1 \mathrm{c}<6.5$ $07 \%$. Las excursiones posprandiales pueden ser normalizadas 0 atenuadas con la dieta adecuada y apoyados por las acciones farmacológicas de antidiabéticos orales y/o inyectados.

Por su mecanismo glucosúrico los iSGLT2 pueden dar resultados favorables en aplanar la curva de glucosa posprandial. Si bien por su efecto de clase todos logran un incremento en la excreción urinaria de glucosa, hay evidencia en estudios comparativos de una mayor eficacia de la canagliflozina para reducir la glucemia posprandial hasta un $10 \%$ más cuando se compara con dapagliflozina en sujetos sanos.

Es posible que los mejores resultados dependan de la inhibición de receptores SGLT1, algunos localizados en la pared del intestino, que disminuyen la absorción de glucosa y que pueden ser el diferenciador de canagliflozina por su efecto inhibitorio en SGLT1 y en SGLT2 ${ }^{20}$.

Recomendaciones

- Se debe iniciar el tratamiento integral desde el diagnóstico, insistiendo en cambios a un estilo de vida saludable. Si hay estabilidad clínica y descontrol leve a moderado iniciar con metformina. Si hay intolerancia a metformina considerar el uso de iSGLT2.

Cuando no se logra un buen control glucémico con metformina y se requiera una combinación farmacológica, adicionar un iSGLT2 puede además de corregir el descontrol hiperglucémico, y colaborar en un mejor control de la presión arterial y del peso corporal.

En todo paciente con diabetes se debe investigar la microalbuminuria, que nos señala riesgo para nefropatía (ND). Está indicado en estos casos la administración de un iSGLT2.

- Los iSGLT2 son una buena opción para reducir la variabilidad de la glucosa y de esta forma reducir también las excursiones posprandiales.

- En el paciente de más de 10 años de evolución suele haber un riesgo incrementado para ECV y aparición o progresión de ND. En estos casos debe considerarse de primera elección la administración de un iSGLT2. Incluso se recomienda cambiar fármacos como iDPP4, glitazonas o sulfonilureas a iSGLT2.

\section{Protección cardiovascular y renal de los iSGLT2}

Existe sólida evidencia que indica que los iSGLT2, además de reducir la glucemia, ofrecen un efecto protector a nivel macrovascular y microvascular derivado de los resultados de los estudios clínicos en los que se evalúa eficacia y seguridad de los iSGLT2 tanto a nivel CV como renal.

El primero de ellos fue el estudio EMPA-REG OUTCOME (Empagliflozin Cardiovascular Outcome Event Trial in Type 2 Diabetes Mellitus Patients), en el que el fármaco estudiado fue la empagliflozina, que se comparó con placebo más cuidados habituales (tratamiento eficaz de hipertensión incluyendo un bloqueador del sistema renina-angiotensina-aldosterona, dislipidemia, antiagregantes plaquetarios y tratamiento no farmacológico). El estudio se realizó en pacientes con DM2 y riesgo CV elevado y su objetivo primario era compuesto (MACE, eventos adversos cardiovasculares mayores: muerte por causas CV, infarto de miocardio no fatal y accidente vascular cerebral no fatal). El estudio demostró que los pacientes que recibieron empagliflozina presentaron significativamente menos muerte $\mathrm{CV}$ con una reducción del riesgo del $38 \%$, de la hospitalización por IC con un $35 \%$ de reducción del riesgo y de muerte por cualquier causa de un $32 \%{ }^{21}$.

Posteriormente se publicaron los resultados del análisis preespecificado de los efectos de este fármaco a nivel microvascular. Se utilizaron datos de los pacientes con TFGe $>30 \mathrm{ml} / \mathrm{min} / 1.73 \mathrm{~m}^{2}$ y los objetivos renales secundarios evaluados fueron: la aparición o empeoramiento de nefropatía (progresión de microalbuminuria, aumento de los niveles de creatinina sérica al doble de los valores iniciales, necesidad de diálisis o muerte de causa renal) e inicio de albuminuria ${ }^{22}$. Los pacientes tratados con empagliflozina presentaron una incidencia significativamente menor de empeoramiento de la ND, respecto del grupo tratado con placebo (12.7 vs. $18.8 \%$ respectivamente; $p<0.001$ ), reducción significativa del $44 \%$ del riesgo de duplicar los niveles de creatinina sérica y $55 \%$ de requerir diálisis ${ }^{22}$.

El programa CANVAS (Canagliflozin Cardiovascular Assessment Study) integró los datos de dos estudios realizados con canagliflozina que incluyeron algo más de 10,000 pacientes con DM2 y elevado riesgo $\mathrm{CV}^{23}$. 
En ambos estudios del programa CANVAS, los pacientes incluidos fueron tratados con canagliflozina o placebo y seguidos durante 188 semanas de mediana. El objetivo primario también fue un objetivo CV compuesto (MACE) y fue significativamente menor en los pacientes tratados con canagliflozina comparado con aquellos tratados con placebo. También se observó una tendencia clara de beneficio renal, aunque no alcanzó significancia estadística; en este sentido, se observó menor progresión de la albuminuria en pacientes tratados con canagliflozina, así como una reducción del $40 \%$ de los eventos renales (reducción de TFGe, necesidad de tratamiento renal sustitutivo o muerte por causa renal) $)^{23-25}$.

En el subestudio CANVAS-R, el riesgo relativo del resultado del desenlace primario compuesto de enfermedad renal en etapa terminal (diálisis, trasplante 0 una TFGe sostenida $<15 \mathrm{ml} / \mathrm{min} / 1.73 \mathrm{~m}^{2}$ ), duplicación de creatinina sérica o muerte por causas renales 0 $\mathrm{CV}$, fue un $30 \%$ menor en el grupo de canagliflozina que en el grupo de placebo ${ }^{26}$.

El estudio DECLARE-TIMI (Dapagliflozin Effect on CardiovascuLAR Events), con dapagliflozina, también tuvo como objetivo primario la posible prevención de eventos CV y tuvo como objetivo secundario la enfermedad renal; en él se incluyeron 17,160 pacientes que fueron seguidos durante 4.2 años de mediana y se observó una mejoría en los resultados renales compuestos (más del $40 \%$ de reducción de la TFGe a $<60 \mathrm{ml} / \mathrm{min} / 1.73 \mathrm{~m}^{2}$, enfermedad renal terminal de nueva aparición o muerte por causa renal o CV). En el global del estudio, la incidencia de resultados renales compuesto fue del $4.3 \%$ en el grupo tratado con dapagliflozina y del $5.6 \%$ en el grupo tratado con placebo (hazard ratio [HR]: 0.76; IC 95\%: 0.67 a 0.87) sin embargo, en este estudio en los pacientes con DM2 que tenían riesgo elevado de ECV, el tratamiento con dapagliflozina, comparado con placebo, no alcanzó resultados estadísticamente significativos en cuanto a un objetivo CV compuesto, aunque sí se observó menor índice de muerte CV o de hospitalizaciones por $\mathrm{IC}^{27}$.

El estudio CREDENCE se realizó en pacientes con DM2 y ERC con albuminuria, y comparó la eficacia de canagliflozina contra placebo y es el primer estudio en tener un objetivo renal primario compuesto. Los pacientes incluidos presentaban TFGe de $30-90 \mathrm{ml} /$ $\mathrm{min} / 1.73 \mathrm{~m}^{2}$ y albuminuria (índice de albumina creatinina $\mathrm{mg} / \mathrm{g}>300$ hasta 5,000 ), en tratamiento a dosis óptimas de fármacos inhibidores del sistema renina-angiotensina. Se trató de un estudio en el que el objetivo primario compuesto fue: enfermedad renal terminal (diálisis, trasplante o TFGe mantenida < $15 \mathrm{ml} / \mathrm{min} / 1.73 \mathrm{~m}^{2}$ ), duplicación de creatinina sérica respecto a valores iniciales o muerte por causa renal $\circ \mathrm{CV}$. El estudio fue interrumpido prematuramente debido a la eficacia observada con canagliflozina después del análisis intermedio por un comité de seguridad y vigilancia. Los resultados mostraron, tras un seguimiento de 2.6 años, que los pacientes tratados con canagliflozina redujeron en $34 \%$ (HR: 0.66; IC 95\%: 0.53 a 0.81; $p<0.001$ ) el riesgo de presentar el objetivo renal compuesto, el riesgo de aumentar a duplicar los niveles de creatinina sérica y el riesgo de muerte por causa renal, y redujeron en un 32\% (HR: 0.68; IC 95\%: 0.54 a 0.86; $p=0.002$ ) el riesgo de enfermedad renal terminal. También se observó una reducción del riesgo de muerte por causa CV, infarto de miocardio o accidente vascular cerebral, así como de hospitalización por IC ${ }^{24}$. El estudio demostró que el uso de canagliflozina, conjuntamente con bloqueo a dosis óptimas del sistema renina-angiotensina-aldosterona en pacientes con DM2 y ERC en estadios 2 a 3, ofrece un significativo beneficio clínico para prevención de eventos renales, así como CV y puede administrarse con seguridad en esta población. Los beneficios absolutos renales son mayores en los pacientes con menor TFGe ${ }^{28}$.

Posteriormente, se realizó el estudio DAPA-CKD (Dapagliflozin and Prevention of Adverse Outcomes in Chronic Kidney Disease), el cual incluyó 4,304 pacientes con DM2 (68\% de la población del estudio), así como pacientes con ERC asociada a otras formas de enfermedad renal (32\% de la población del estudio), con TFGe de $25-75 \mathrm{ml} / \mathrm{min} / 1.73 \mathrm{~m}^{2}$ e índice de albúmina-creatinina de $200-5,000$, que fueron tratados con dapagliflozina o placebo. Los resultados mostraron que tanto en pacientes con ND como en aquellos con nefropatía de otro origen, el riesgo del objetivo renal compuesto (descenso sostenido de al menos el $50 \%$ de la TFGe, ERC en estadio terminal o muerte por causa renal o $\mathrm{CV}$ ) se redujo significativamente en los pacientes tratados con dapagliflozina comparados con los tratados con placebo (HR: 0.61; IC 95\%: 0.51 a $0.72 ; p<0.001)^{29}$.

Un metaanálisis que incluyó 48 estudios que aportaron datos de 58,165 pacientes tratados con iSGLT2 demostró los efectos benéficos renales de estos al reducir el riesgo de desarrollar albuminuria (riesgo relativo [RR]: 0.49 ; IC 95\%: 0.33 a 0.73 ; $p<0.001$ ) y de enfermedad renal terminal, comparado con placebo o con otros antidiabéticos (RR: 0.73; IC 95\%: 
0.58 a $0.93 ; p=0.012)^{30}$. Un segundo metaanálisis, esta vez realizado con los estudios EMPA-REG, CANVAS y DECLARE-TIMI, que estratificó los pacientes $(n=34,322)$ según su nivel de ERC, demostró que los iSGLT2 reducían más el riesgo de desenlaces renales adversos (empeoramiento de la insuficiencia renal, ERC terminal o muerte por causa renal) en aquellos con una TFGe de 30-60 $\mathrm{ml} / \mathrm{min} / 1.73 \mathrm{~m}^{2}(0.55$; IC 95\%: 0.48 a $0.64 ; p<0.0001)^{31}$. Finalmente, un tercer metaanálisis de los estudios EMPA-REG, programa CANVAS, CREDENCE y DECLARE-TIMI-58 que incluyó un total de 38,723 pacientes, también constató los beneficios del tratamiento con iSGLT2, con una reducción del riesgo de diálisis, trasplante o muerte por causa CV o renal (RR: 0.67; IC 95\%: 0.52 a $0.87 ; p=0.0019$ ), de enfermedad renal terminal (RR: 0.65; IC 95\%: 0.53 a 0.81; $p<0.0001$ ), así como de desarrollar enfermedad renal aguda (RR: 0.75 ; IC 95\%: 0.66 a $0.85 ; p<0.0001)^{32}$.

Recomendación

- En función de los resultados de los estudios y de las recomendaciones de las principales guías internacionales, se recomienda como primera línea de tratamiento de pacientes con DM2 y ERC el uso de iSGLT2, habitualmente en combinación con metformina, si su TFGe es superior a $30 \mathrm{ml} /$ $\mathrm{min} / \mathrm{m}^{2}$.

- A la fecha de esta publicación, los iSGLT2 que han demostrado eficacia en retrasar daño renal en estudios cuyo objetivo primario es un compuesto de progresión renal son la canagliflozina y la dapagliflozina. Se encuentra en curso un estudio similar con empagliflozina (EMPAKID$\mathrm{NEY}$ ), que evaluará específicamente el efecto de este iSGLT2 en una amplia población de pacientes con ND en DM1 y DM2, con otras formas de nefropatía, con TFGe desde $20 \mathrm{ml} / \mathrm{min} / 1.73 \mathrm{~m}^{2} \mathrm{y}$ con o sin albuminuria.

- Los pacientes con DM tienen habitualmente riesgo CV aumentado, esté presente o no clínicamente. Por lo anterior, se considera igualmente apropiado el empleo de un iSGLT2 para protección $\mathrm{CV}$, tal como se ha descrito en los estudios EMPA-REG, CANVAS, DECLARE-TIMI, CREDENCE y DAPA-CKD 21,22,24,27-29.

- Los pacientes con DM, particularmente los que han estado con descontrol crónico, pueden tener un riesgo mayor para ECV, nefropatía y retinopatía. Los diferentes estudios sobre iSGLT2 tienen evidencia de que su administración ejerce efectos benéficos y puede retrasar o prevenir complicaciones microvasculares y macrovasculares

- Con base en los resultados del estudio CREDENCE y para lograr reducir la progresión de la ND se puede iniciar tratamiento con canagliflozina en pacientes con TFGe desde $30 \mathrm{ml} / \mathrm{min} / 1.73$ $\mathrm{m}^{2}$. En estos casos se puede mantener la canagliflozina como tratamiento aun cuando pueda bajar más la TFGe ${ }^{10,33}$. Por otra parte, el estudio DAPA-CKD incluyó pacientes con TFGe desde $25 \mathrm{ml} / \mathrm{min} / 1.73 \mathrm{~m}^{2}$ y que continuaron también usando el fármaco hasta el desenlace renal primario.

- De acuerdo con autorizaciones regulatorias y con la evidencia actual, en pacientes con TFG $<30 \mathrm{ml} / \mathrm{min} / 1.73 \mathrm{~m}^{2}$ no debe iniciarse tratamiento con un iSGLT2, aunque de forma individualizada puede continuarse aun en pacientes con una filtración menor y hasta inicio de diálisis, tal y como se hizo en los estudios CREDENCE y DAPA-CKD.

\section{Desenlaces renales con AR-GLP1}

Como se explicó, los iSGLT2 tienen una sólida evidencia en la reducción de la insuficiencia cardíaca y de la progresión de enfermedad renal la progresión de enfermedad renal ${ }^{4}$. Por otra parte, en estudios fase III en los que se evaluaba como objetivo primario la seguridad y el efecto de los AR-GLP1 sobre desenlaces CV en sujetos con DM2, también se observó como parte de los objetivos secundarios su eficacia en la protección renal. Estos beneficios renales fueron parte de los resultados de objetivos secundarios, sin embargo, hoy en día se encuentran en curso evaluaciones del efecto de AR-GLP1 en la enfermedad renal como objetivo primario.

El estudio LEADER (Liraglutide Effect and Action in Diabetes: Evaluation of Cardiovascular Outcome Results), que comparó liraglutida comparada con placebo, incluyó 9,340 pacientes con DM2 y ECV o riesgo CV elevado (de los cuales 220 presentaban TFGe $15-30 \mathrm{ml} / \mathrm{min} / 1.73 \mathrm{~m}^{2}$ ), que fueron seguidos durante 3.8 años $^{34}$. Además de la reducción de HbA1c y del peso, se observó retraso en la progresión de la ND en un $22 \%$ en el grupo de liraglutida en comparación con el grupo placebo, principalmente debido a una reducción de casos nuevos con albuminuria significativa ${ }^{4,35}$. 
El estudio SUSTAIN-6 (Semaglutide in Subjects With Type 2 Diabetes) incluyó 3,297 pacientes, con riesgo CV incrementado, que fueron tratados con semaglutida o placebo y cuyos resultados fueron similares a lo ya descrito, de forma que los pacientes tratados con semaglutida presentaron menor progresión de ND que los tratados con placebo (HR: 0.78; IC 95\%: 0.67 a 0.92; $p=0.003)^{36}$. En este estudio el $10.7 \%$ de los pacientes presentaban únicamente ERC y el $13.4 \%$ ERC y ECV 4 .

En el estudio ELIXA (Evaluation of Lixisenatide in Acute Coronary Syndrome) se incluyeron 6,068 pacientes con DM2 y síndrome coronario agudo, y a diferencia de los estudios LEADER y SUSTAIN-6, el control glucémico y la reducción del peso fueron menos marcadas comparado con el grupo tratado con placebo, así como los beneficios renales evaluados con el porcentaje de cambio del índice albumina-creatinina (24 vs. $34 \% ; p=0.004)^{37}$.

El estudio EXSCEL (Exenatide Study of Cardiovascular Event Lowering) valoró la eficacia de exenatida de acción prolongada administrada como inyectable subcutáneo e incluyó 14,752 pacientes con o sin ECV, demostrando una reducción del $40 \%$ en el objetivo renal compuesto en un análisis ajustado ${ }^{38}$.

Reiteramos, hasta el momento actual todos los estudios con AR-GLP1 que han mostrado beneficio renal lo han hecho como objetivo secundario, por lo que la evidencia es indirecta y no suficientemente sólida como para generar una indicación específica en esta población de enfermos, para el manejo de la ND. En estudios en curso se responderá esta pregunta próximamente.

Recomendaciones

- Tanto los AR-GLP-1 como los iSGLT2 reducen los eventos CV compuestos de forma similar, sin embargo, y de acuerdo con las guías KDIGO, los iSGLT2 tienen evidencia más sólida en la reducción de la IC y de progresión de enfermedad renal ${ }^{4}$.

- Se recomiendan los AR-GLP1 que hayan demostrado beneficios CV y en forma secundaria beneficios renales, tales como liraglutida, semaglutida y dulaglutida ${ }^{4}$.

- En pacientes con DM2, ERC y TFGe $\geq 30 \mathrm{ml} / \mathrm{min} / 1.73 \mathrm{~m}^{2}$, con la evidencia actual es preferible el uso de iSGLT-2 que el de AR-GLP1 como tratamiento inicial hipoglucemiante y protector orgánico, juntamente con metformina ${ }^{4}$, reservándose los AR-GLP1 como alternativa en caso de intolerancia o mal control glucémico con iSGLT2 más metformina ${ }^{4}$.

\section{Otros efectos de los iSGLT y AR-GLP1}

Además del efecto hipoglucemiante, los iSGLT2 tienen efectos pleiotrópicos entre los que destaca la reducción del peso, de la presión arterial, disminución del ácido úrico sérico y de marcadores inflamatorios ${ }^{12}$.

En general, se ha constatado que los iSGLT2 reducen la presión sistólica en 3.4 a $5.4 \mathrm{mmHg}$ y la diastólica en 1.5 a $2.2 \mathrm{mmHg}$ de mediana ${ }^{39}$. Los pacientes con DM2 cuya presión no esté controlada son los que obtendrán mayor reducción de la presión sistólica con el tratamiento con iSGLT2 ${ }^{39}$.

Un análisis en el que se utilizaron datos de pacientes incluidos en cuatro estudios clínicos de fase III con ERC en estadio 3 y DM2 tratados con canagliflozina demostró la reducción de HbA1c $(-0.38$ y $-0.47 \% ; p<0,001)$, del peso $(-1.6$ y $-1.9 ; p<0.001)$ y de la presión sistólica $(-2.8$ y $-4.4 \mathrm{mmHg} ; p<0.01)$ en los pacientes tratados con canagliflozina $100 \mathrm{y}$ $300 \mathrm{mg}$ respectivamente ${ }^{40}$.

Por su parte, los AR-GLP1 también reducen la presión arterial al inducir diuresis y natriuresis, así como mejoran la dislipidemia y reducen el peso ${ }^{41}$.

Recomendación

- En general, ni los iSGLT2 ni los AR-GLP1 son fármacos que deban utilizarse como tratamiento de la obesidad o reducción de peso. Sin embargo, la liraglutida (AR-GLP1) está autorizada para el tratamiento de la obesidad por la FDA.

- En un paciente con diabetes, ERC y obesidad se deberá tener presente que los iSGLT2 son agentes que además de sus propiedades hipoglucemiantes y nefroprotectoras, favorecen también una reducción del peso.

\section{Paciente trasplantado renal}

Debido al uso del paciente trasplantado de riñón de inmunosupresores que pueden ser inductores de hiperglucemia, particularmente esteroides, así como tacrolimús, el riesgo de presentar diabetes postrasplante (DMPT) está presente y siempre latente. En forma paralela, se incrementa el riesgo de infecciones, de hiperlipidemia y de hipertensión, lo que a su vez aumenta la morbimortalidad y reduce la supervivencia del órgano injertado ${ }^{42}$.

En el paciente trasplantado que presente DM y que su ERC fue secundaria a ND o bien en el que desarrolle 
DMPT se promoverán, al igual que con cualquier otro paciente con DM no trasplantado, modificaciones del estilo de vida y dietéticas encaminadas a la reducción de peso, entre otras, con el fin de reducir el mayor riesgo de complicaciones microvasculares y macrovasculares ${ }^{42}$.

Las guías KDIGO recomiendan el uso de metformina en pacientes trasplantados que tengan TFGe $\geq 30 \mathrm{ml} / \mathrm{min} / 1.73 \mathrm{~m}^{2}$ y aumentar la frecuencia de monitorización cuando la TFGe $<60 \mathrm{ml} / \mathrm{min} / 1.73 \mathrm{~m}^{24}$.

Los iSGLT2 son fármacos que potencialmente podrían utilizarse en pacientes con DM postrasplantados 0 con DMPT de novo debido a sus efectos específicos, como pérdida de peso, bajo riesgo de hipoglucemia, renoprotección y cardioprotección, así como por reducir la incidencia y el número de ingresos debidos a $\mathrm{IC}^{4}$. Sin embargo, la evidencia disponible respecto a la eficacia y seguridad de los iSGLT2 en pacientes trasplantados es escasa.

Los AR-GLP1 pueden ser potencialmente utilizados en personas con DM trasplantados o con DMPT de novo, aunque por igual no existe la suficiente evidencia sobre su seguridad, eficacia ni efectos microvasculares y/o macrovasculares a largo plazo ${ }^{4,42}$.

Recomendación

- Tanto los iSGLT como los AR-GLP-1 en un paciente trasplantado renal no están contraindicados formalmente, aunque se carece de evidencias clínicas controladas y no existe una indicación específica para su uso, por lo que se deberá evaluar individualmente cada caso.

\section{Seguridad}

Los pacientes con DM y ERC son frágiles y pueden presentar más efectos adversos con diversos fármacos, por lo que en general, es preciso ajustar la dosis de estos.

Se han descrito algunos efectos adversos relacionados con los iSGLT2, como la presencia de infecciones genitourinarias/micóticas. En algunos estudios se observó incremento en el riesgo de fracturas y de amputaciones periféricas, sin embargo estos hallazgos no se han corroborado en estudios posteriores incluso con las mismas formulaciones ${ }^{32}$. Los efectos beneficiosos de los iSGLT2 a nivel CV y renal superan los posibles efectos adversos, especialmente en los pacientes con riesgo $\mathrm{CV}$ elevado ${ }^{43}$.

\section{Infecciones genitourinarias/micóticas}

En los principales estudios CV realizados con los iSGLT2 (EMPA-REG OUTCOME, CANVAS,
DECLARE-TIMI-58) se observó un aumento ligero pero significativo de infecciones micóticas (balanitis y vulvovaginitis) al comparar los pacientes tratados con iSGLT2 con los tratados con placebo ${ }^{43,44}$. En la mayoría de los casos, estas infecciones micóticas son leves y no precisan interrumpir el tratamiento; se asocian a infecciones micóticas previas o al uso de tratamiento estrogénico ${ }^{39,44}$. Se pueden tratar con antifúngicos tópicos ${ }^{4,39}$, recomendándose la higiene diaria para reducir el riesgo de infecciones micóticas ${ }^{4}$.

\section{Amputaciones}

El incremento del riesgo de fracturas y de amputaciones de la falanges distales del miembro inferior que se observó en el estudio CANVAS con canagliflozina no se ha confirmado en otros estudios clínicos ni en estudios observacionales realizados con canagliflozina o con los otros iSGLT2 (dapagliflozina y empagliflozina) $)^{43}$. Un estudio de vida real, en el que se comparó el riesgo de hospitalización por IC y la incidencia de amputaciones de miembros inferiores por debajo de la rodilla en más de 700,000 pacientes tratados con canagliflozina, otros iSGLT2 u otros hipoglucemiantes no iSGLT2, no se observó aumento del riesgo de amputaciones en los pacientes tratados con canaglifozina comparado con los otros tratamientos ${ }^{45}$. De hecho, la FDA eliminó la advertencia previa sobre amputaciones que había emitido sobre canagliflozina ${ }^{25}$.

Las guías KDIGO indican que los cuidados preventivos rutinarios de los pies y una hidratación adecuada pueden reducir el riesgo de complicaciones, en los mismo estudios no se ha demostrado una mayor incidencia de infecciones bacterianas en vías urinarias ${ }^{4}$.

\section{Fracturas}

En el programa CANVAS, la tasa de todas las fracturas fue mayor con canagliflozina que con placebo (15.4 frente a 11.9 participantes con fractura por 1,000 personas-año; HR: 1.26; IC 95\%: 1.04 a 1.52) ${ }^{39}$. Sin embargo, este aumento de riesgo no se observó en los pacientes incluidos en el estudio CANVAS-R o en estudios con otros iSGLT2. El aumento del riesgo de fracturas según este estudio ocurrió principalmente en las primeras semanas de tratamiento ${ }^{39}$. En un metaanálisis que incluyó 38 estudios clínicos de pacientes tratados con canagliflozina, dapagliflozina y empagliflozina y que representaron un total de más de 30,000 pacientes seguidos entre 24 y 160 semanas, no se 
observaron diferencias en el índice de fracturas en los pacientes tratados con iSGLT2 comparado con los controles (1.59 vs. $1.56 \%$ ) y la incidencia de fracturas fue similar entre los tres iSGLT2 valorados ${ }^{46}$.

\section{Cetoacidosis diabética}

La cetoacidosis diabética (CAD) es una complicación infrecuentemente descrita con iSGLT2 ${ }^{4,12}$. De hecho, en el estudio EMPA-REG-OUTCOME, la proporción de pacientes con CAD fue muy baja tanto en los pacientes tratados con empagliflozina como en los tratados con placebo $(0.09$ vs. $0.04 \%$, respectivamente) y sin diferencias significativas entre ambos grupos $^{21,39}$. El programa CANVAS también detectó una incidencia de CAD muy baja en los pacientes tratados con canagliflozina y en los tratados con placebo ( 0.6 vs. 0.3 eventos por 1,000 pacientes-año, respectivamente) $)^{39}$. El estudio DAPA-CKD no observó un solo caso de CAD en los pacientes que recibieron dapagliflozina $^{29}$. En caso de que se presentase esta infrecuente complicación, el tratamiento de la CAD será como habitualmente se realiza, con la administración de líquidos endovenosos, insulina y electrolitos.

A pesar de ser muy infrecuente, las fichas técnicas de los iSGLT2 comercializados advierten del riesgo de CAD en pacientes con DM2, misma que puede presentarse de forma atípica y que debe considerarse en el caso de síntomas inespecíficos como náuseas, vómitos, anorexia, dolor abdominal, sed excesiva, dificultad respiratoria, confusión, fatiga o somnolencia inusuales. Si la persona diabética presenta CAD durante el tratamiento con iSGLT2, estos deberán interrumpirse $\mathrm{e}^{39}$.

Como es claramente conocido, la CAD es una complicación más frecuentemente observada en DM1. Hasta el momento actual los iSGLT2 no han sido aprobados en DM1.

\section{Depleción de volumen}

Los iSGLT2 producen diuresis osmótica y natriuresis significativa, mecanismos por los cuales se reduce la presión arterial. Esta diuresis es beneficiosa en pacientes con hipertensión no controlada. Sin embargo, en personas de edad avanzada con enfermedad renal o en pacientes que estén siendo tratados con diuréticos de asa, este efecto pudiera provocar mareos posturales, hipotensión ortostática y deshidratación.
En el estudio EMPA-REG OUTCOME ${ }^{21}$, los efectos adversos relacionados con la depleción de volumen fueron raros y su frecuencia fue similar en los grupos de empagliflozina y placebo (5.1 vs. $4.9 \%$, respectivamente). Por el contrario, en CANVAS ${ }^{23}$ la depleción de volumen fue más común en el grupo de canagliflozina que en el de placebo (26 vs. 18.5 eventos por 1,000 personas/año; $p=0.009$ ). En el estudio DAPACKD la presencia de depleción de volumen sintomática fue también infrecuente y no significativamente diferente entre el grupo que recibió dapagliflozina y el grupo placebo ${ }^{29}$.

Los efectos adversos más frecuentes con el uso de AR-GLP1 son los gastrointestinales (náuseas, vómitos y diarrea), mismos que son dependientes de la dosis y pueden variar entre los distintos AR-GLP14; generalmente se autolimitan después de dos a cuatro semanas de uso y disminuye su frecuencia con el inicio escalonado de la dosificación. Adicionalmente, se ha observado que los AR-GLP1 aumentan la frecuencia cardiaca, aunque no se ha identificado ningún trastorno del ritmo cardiaco $0^{4,10}$.

En pacientes con historia previa de colelitiasis o pancreatitis, los AR-GLP1 se deben usar con precaución, ya que se ha observado aumento del riesgo de problemas biliares agudos ${ }^{10}$. Están contraindicados en pacientes con historia personal o familiar de cáncer de tiroides o de neoplasia endocrina múltiple tipo $2^{4,10}$.

Recomendaciones

- Los iSGLT2 son fármacos seguros en pacientes con DM2 con o sin ERC. En adición a su efecto hipoglucemiante descrito, se ha demostrado beneficio en protección CV y en protección renal desde etapas tempranas de la nefropatía diabética con microalbuminuria como única manifestación hasta estadios avanzados con TFGe de 25 a $30 \mathrm{ml} / \mathrm{min} / 1.73 \mathrm{~m}^{2} \mathrm{sc}$.

- No existe un efecto de clase ni suficiente evidencia que indique que se aumenta el riesgo de amputaciones y/o fracturas con los iSGLT2.

- Existe asociación poco frecuente entre el uso de iSGLT2 y la CAD. Los pacientes con mayor riesgo son los que cursan DM1 y DM2 y que dependen de insulina para un buen control. Por lo anterior se deberá tener una vigilancia clínica más estrecha y monitorizar niveles de cetonas en orina. Los iSGLT2 no están aprobados para su uso clínico en DM1.

- La canagliflozina, la empagliflozina y la dapagliflozina son seguras en pacientes con albuminuria y ERC moderada ${ }^{12,21,22,24,29}$. 


\section{Financiamiento}

La realización de este trabajo de consenso fue auspiciada por un donativo no restringido al grupo de trabajo por Janssen, México. Las sugerencias presentadas en el siguiente documento son opinión de los autores y no representan una opinión o recomendación oficial de alguna sociedad o compañía editorial.

\section{Conflicto de intereses}

Los autores declaran que no existe conflicto de intereses.

\section{Responsabilidades éticas}

Protección de personas y animales. Los autores declaran que para esta investigación no se han realizado experimentos en seres humanos ni en animales.

Confidencialidad de los datos. Los autores declaran que en este artículo no aparecen datos de pacientes.

Derecho a la privacidad y consentimiento infor-

mado. Los autores declaran que en este artículo no aparecen datos de pacientes.

\section{Bibliografía}

1. Méndez-Durán A, Ignorosa-Luna MH, Pérez-Aguilar G, Rivera-Rodríguez FJ, González-Izquierdo JJ, Dávila-Torres J. Estado actual de las terapias sustitutivas de la función renal en el Instituto Mexicano del Seguro Social. Rev Med Inst Mex Seguro Soc. 2016;54(5):588-93.

2. Méndez-Durán A. Evolution of renal replacement therapy in Mexico in the last 10 years. Nefrologia (Engl Ed). 2021;41(1):82-3.

3. American Diabetes Association. 11. Microvascular complications and foot care: Standards of Medical Care in Diabetes-2021. Diabetes Care. 2021;44(Suppl 1):S151-S167.

4. Kidney Disease: Improving Global Outcomes (KDIGO) Diabetes Work Group. KDIGO 2020 Clinical Practice Guideline for Diabetes Management in Chronic Kidney Disease. Kidney Int. 2020;98(4S):S1-S115.

5. Aldrete-Velasco JA, Chiquete E, Rodríguez-García JA, Rincón-Pedrero R, Correa-Rotter R, García-Peña R, et al. Mortalidad por enfermedad renal crónica y su relación con la diabetes en México. Med Int Mex. 2018;34(4):536-50.

6. Cosentino F, Grant PJ, Aboyans V, Bailey CJ, Ceriello A, Delgado V, et al.; ESC Scientific Document Group. 2019 ESC Guidelines on diabetes, pre-diabetes, and cardiovascular diseases developed in collaboration with the EASD. Eur Heart J. 2020;41(2):255-323.

7. Lin $X, X u Y$, Pan $X, X u$ J, Ding $Y$, Sun $X$, et al. Global, regional, and national burden and trend of diabetes in 195 countries and territories: an analysis from 1990 to 2025. Sci Rep. 2020;10(1):14790.

8. Instituto Nacional de Estadística y Geografía e Instituto Nacional de Salud Pública. Encuesta Nacional de Nutrición y Salud 2018. Presentación de resultados [Internet]. México: Instituto Nacional de Estadística y Geografía e Instituto Nacional de Salud Pública [último acceso: abril 2021]. Disponible en: https://ensanut.insp.mx/encuestas/ensanut2018/ doctos/informes/ensanut_2018_presentacion_resultados.pdf

9. International Diabetes Federation. IDF Diabetes Atlas. Eight edition 2017 [internet]. International Diabetes Federation; 2017 [último acceso: abril 2021]. Disponible en: http://fmdiabetes.org/wp-content/uploads/2018/03/ IDF-2017.pdf

10. Li J, Albajrami O, Zhuo M, Hawley CE, Paik JM. Decision Algorithm for prescribing SGLT2 inhibitors and GLP-1 receptor agonists for diabetic kidney disease. Clin J Am Soc Nephrol. 2020;15(11):1678-88.
11. Informe al Ejecutivo Federal y al Congreso de la Unión sobre la Situación Financiera y los Riesgos del Instituto Mexicano del Seguro Social 20182019 [Internet]. Instituto Mexicano del Seguro Social [último acceso: junio 2021]. Disponible en: http://www.imss.gob.mx/sites/all/statics/pdf/ informes/20182019/21-InformeCompleto.pdf

12. Scheen AJ. Efficacy and safety profile of SGLT2 inhibitors in patients with type 2 diabetes and chronic kidney disease. Expert Opin Drug Saf. 2020;19(3):243-56.

13. Garber AJ, Handelsman Y, Grunberger G, Einhorn D, Abrahamson MJ, Barzilay Jl, et al. Consensus statement by the American Association of Clinical Endocrinologists and American College of Endocrinology on the comprehensive type 2 diabetes management algorithm - 2020 executive summary. Endocr Pract. 2020;26(1):107-39.

14. American Diabetes Association. Standards of Medical Care in Diabetes-2022. Diabetes Care. 2022;43(Suppl 1):S1-204.

15. Hsia DS, Grove O, Cefalu WT. An update on sodium-glucose co-transporter-2 inhibitors for the treatment of diabetes mellitus. Curr Opin Endocrinol Diabetes Obes. 2017;24(1):73-9.

16. Miranda Fernández-Santos $C$, Egocheaga Cabello MI. Guía práctica de uso de los iSGLT2 en diabetes mellitus tipo 2 [Internet]. Sociedad Española de Médicos Generales y de Familia; 2020 [último acceso: abril 2021]. Disponible en: https://www.semg.es/images/documentos/guia_ uso_iSGLT2.pdf

17. Polidori D, Sha S, Mudaliar S, Ciaraldi TP, Ghosh A, Vaccaro N, et al. Canagliflozin lowers posprandial glucose and insulin by delaying intestinal glucose absorption in addition to increasing urinary glucose excretion: Results of a randomized, placebo-controlled study. Diabetes Care. 2013;36:2154-61.

18. Takebayashi K, Hara K, Terasawa T, Naruse R, Suetsugu M, Tsuchiya T, et al. Effect of canagliflozin on circulating active glp-1 levels in patients with type 2 diabetes: A randomized trial. Endocr J. 2017;64:923-31.

19. Toyama T, Neuen BL, Jun M, Ohkuma T, Neal B, Jardine MJ, et al. Effect of SGLT2 inhibitors on cardiovascular, renal and safety outcomes in patients with type 2 diabetes mellitus and chronic kidney disease: A systematic review and meta-analysis. Diabetes Obes Metab. 2019;21(5):1237-50.

20. Sha S, Polidori D, Farrell K, Ghosh A, Natarajan J, Vaccaro N, et al. Pharmacodynamic differences between canagliflozin and dapagliflozin: results of a randomized, double-blind, crossover study. Diabetes Obes Metab. 2015:17(2):188-97.

21. Zinman B, Wanner C, Lachin JM, Fitchett D, Bluhmki E, Hantel S, et al. Empagliflozin, cardiovascular outcomes, and mortality in type 2 diabetes. N Engl J Med. 2015; 373:2117-28.

22. Wanner C, Inzucchi SE, Lachin JM, Fitchett D, von Eynatten M, Mattheus $\mathrm{M}$, et al. Empagliflozin and progression of kidney disease in type 2 diabetes. N Engl J Med. 2016;375:323-34.

23. Neal B, Perkovic V, Mahaffey KW, de Zeeuw D, Fulcher G, Erondu N, et al. Canagliflozin and cardiovascular and renal events in type 2 diabetes. N Engl J Med. 2017; 377:644-57.

24. Perkovic V, Jardine MJ, Neal B, Bompoint S, Heerspink HJL, Charytan DM, et al. Canagliflozin and renal outcomes in type 2 diabetes and nephropathy. N Engl J Med. 2019;380:2295-306.

25. FDA removes Boxed Warning about risk of leg and foot amputations for the diabetes medicine canagliflozin (Invokana, Invokamet, Invokamet XR) [Internet]. EE.UU.: Food and Drug Administration [publicado: 09/2/2020]. Disponible en: https://www.fda.gov/drugs/drug-safety-and-availability/ fda-removes-boxed-warning-about-risk-leg-and-foot-amputations-diabetes-medicine-canagliflozin

26. Perkovic V, de Zeeuw D, Mahaffey KW, Fulcher G, Erondu N, Shaw W, et al. Canagliflozin and renal outcomes in type 2 diabetes: results from the CANVAS Program randomised clinical trials. Lancet Diabetes Endocrinol. 2018;6(9):691-704.

27. Wiviott SD, Raz I, Bonaca MP, Mosenzon O, Kato ET, Cahn A, et al. Dapagliflozin and cardiovascular outcomes in type 2 diabetes. $N$ Engl $J$ Med. 2019;380:347-57.

28. Jardine MJ, Zhou Z, Mahaffey KW, Oshima M, Agarwal R, Bakris G, et al.; CREDENCE Study Investigators. Renal, cardiovascular, and safety outcomes of canagliflozin by baseline kidney function: A secondary analysis of the CREDENCE randomized trial. J Am Soc Nephrol. 2020;31(5):1128-39.

29. Heerspink HJL, Stefánsson BV, Correa-Rotter R, Chertow GM, Greene T, Hou FF, et al. Dapagliflozin in patients with chronic kidney disease. N Engl J Med. 2020;383:1436-46.

30. Bae JH, Park EG, Kim S, Kim SG, Hahn S, Kim NH. Effects of sodium-glucose cotransporter 2 inhibitors on renal outcomes in patients with type 2 diabetes: A systematic review and meta-analysis of randomized controlled trials. Sci Rep. 2019;9(1):13009.

31. Zelniker TA, Wiviott SD, Raz I, Im K, Goodrich EL, Bonaca MP, et al. SGLT2 inhibitors for primary and secondary prevention of cardiovascular and renal outcomes in type 2 diabetes: a systematic review and meta-analysis of cardiovascular outcome trials. Lancet. 2019;393(10166):31-9.

32. Neuen BL, Young T, Heerspink HJL, Neal B, Perkovic V, Billot L, et al. SGLT2 inhibitors for the prevention of kidney failure in patients with type 2 diabetes: a systematic review and meta-analysis. Lancet Diabetes Endocrinol. 2019;7(11):845-54. 
33. Saisho Y. SGLT2 inhibitors: The star in the treatment of type 2 diabetes? Diseases. 2020;8(2):14

34. Marso SP, Daniels GH, Brown-Frandsen K, Kristensen P, Mann JF, Nauck MA, et al.; LEADER Steering Committee; LEADER Trial Investigators. Liraglutide and cardiovascular outcomes in type 2 diabetes. N Engl J Med. 2016;375(4):311-22

35. Mann JFE, Ørsted DD, Brown-Frandsen K, Marso SP, Poulter NR, Rasmussen S, et al.; LEADER Steering Committee and Investigators. Liraglutide and renal outcomes in type 2 diabetes. N Engl J Med. 2017;377(9):839-48.

36. Marso SP, Bain SC, Consoli A, Eliaschewitz FG, Jódar E, Leiter LA, et al.; SUSTAIN-6 Investigators. Semaglutide and cardiovascular outcomes in patients with type 2 diabetes. N Engl J Med. 2016:375(19):1834-44.

37. Pfeffer MA, Claggett B, Diaz R, Dickstein K, Gerstein HC, Køber LV et al.; ELIXA Investigators. Lixisenatide in patients with type 2 diabetes and acute coronary syndrome. N Engl J Med. 2015;373(23):2247-57.

38. Holman RR, Bethel MA, Mentz RJ, Thompson VP, Lokhnygina $Y$ Buse JB, et al.; EXSCEL Study Group. Effects of Once-weekly exenatide on cardiovascular outcomes in type 2 diabetes. $\mathrm{N}$ Engl $\mathrm{J}$ Med. 2017:377(13):1228-39.

39. Lupsa BC, Inzucchi SE. Use of SGLT2 inhibitors in type 2 diabetes: weighing the risks and benefits. Diabetologia. 2018;61(10):2118-25.

40. Yamout H, Perkovic V, Davies M, Woo V, de Zeeuw D, Mayer C, et al. Efficacy and safety of canagliflozin in patients with type 2 diabetes and stage 3 nephropathy. Am J Nephrol. 2014;40(1):64-74
41. Greco EV, Russo G, Giandalia A, Viazzi F, Pontremoli R, De Cosmo S. GLP-1 receptor agonists and kidney protection. Medicina (Kaunas). 2019;55(6):233.

42. Ahmed SH, Biddle K, Augustine T, Azmi S. Post-transplantation diabetes mellitus. Diabetes Ther. 2020;11(4):779-801.

43. Scheen AJ. An update on the safety of SGLT2 inhibitors. Expert Opin Drug Saf. 2019;18(4):295-311.

44. Nakhleh A, Zloczower M, Gabay L, Shehadeh N. Effects of sodium glucose co-transporter 2 inhibitors on genital infections in female patients with type 2 diabetes mellitus- Real world data analysis. J Diabetes Complications. 2020;34(7):107587.

45. Ryan PB, Buse JB, Schuemie MJ, DeFalco F, Yuan Z, Stang PE, et al. Comparative effectiveness of canagliflozin, SGLT2 inhibitors and nonSGLT2 inhibitors on the risk of hospitalization for heart failure and amputation in patients with type 2 diabetes mellitus: A real-world meta-analysis of 4 observational databases (OBSERVE-4D). Diabetes Obes Metab. 2018;20(11):2585-97

46. Tang HL, Li DD, Zhang JJ, Hsu YH, Wang TS, Zhai SD, et al. Lack of evidence for a harmful effect of sodium-glucose co-transporter 2 (SGLT2) inhibitors on fracture risk among type 2 diabetes patients: a network and cumulative meta-analysis of randomized controlled trials. Diabetes Obes Metab. 2016;18(12):1199-20. 